\title{
Ultrasound properties of articular cartilage immediately after osteochondral grafting surgery: in cases of traumatic cartilage lesions and osteonecrosis
}

\author{
Hiroshi Kuroki · Yasuaki Nakagawa $\cdot$ Koji Mori · \\ Masahiko Kobayashi · Shinichiro Nakamura • \\ Kohei Nishitani · Takaaki Shirai · Takashi Nakamura
}

Received: 28 December 2007/Accepted: 2 July 2008/Published online: 7 August 2008

(c) The Author(s) 2008. This article is published with open access at Springerlink.com

\begin{abstract}
Although osteochondral grafting surgery is believed to replace damaged cartilage with healthy-looking normal cartilage, no study focuses on ultrasound quantification of those cartilage immediately after the surgery. It is unknown whether the ultrasound properties of damaged cartilage from trauma or osteonecrosis are same with each other. We have examined ultrasound properties of damaged cartilage, adjacent intact cartilage and plug cartilage during osteochondral grafting surgery for 15 knees of 15 patients, mean age of 43.4 (range, 14-61) years. Results showed that signal intensities of ultrasound (a measure of superficial cartilage integrity) of intact cartilage, damaged cartilage, and plug cartilage were 1.91 (arbitrary unit), 0.40, and 2.13, respectively, in 11 knees of 11 patients of traumatic cartilage lesions. Interval between signals of ultrasound (a parameter related to thickness) were 2.79 ( $\mu \mathrm{s}), 1.90$, and 2.85 , respectively. The signal intensity and the interval between signals of plug cartilage were $533 \%(P<0.01)$ and $150 \%(P<0.01)$ of damaged cartilage, respectively.
\end{abstract}

H. Kuroki

Department of Physical Therapy, Human Health Sciences, Graduate School of Medicine, Kyoto University,

53 Kawahara-Cho, Shogoin, Sakyo-Ku, Kyoto 606-8507, Japan

e-mail: kuro@hs.med.kyoto-u.ac.jp

Y. Nakagawa $(\bowtie) \cdot$ M. Kobayashi · S. Nakamura .

K. Nishitani - T. Shirai · T. Nakamura

Department of Orthopaedic Surgery, Graduate School

of Medicine, Kyoto University, 54 Kawahara-Cho,

Shogoin, Sakyo-Ku, Kyoto 606-8507, Japan

e-mail: ynaka@kuhp.kyoto-u.ac.jp

K. Mori

Department of Applied Medical Engineering Science, Graduate School of Medicine, Yamaguchi University,

2-16-1 Tokiwadai, Ube, Yamaguchi 755-8611, Japan
In four knees of four patients of osteonecrosis, the signal intensities of intact cartilage, damaged cartilage, and plug cartilage were $1.25,0.30$, and 1.39 , respectively. The interval between signals were 2.36, 2.00, and 2.69, respectively. The signal intensity and the interval between signals of plug cartilage were $463 \%(P<0.01)$ and $135 \%$ $(P<0.05)$ of damaged cartilage. We conclude that ultrasound properties of the damaged cartilage from trauma or osteonecrosis did not differ a lot especially in the late stages of osteonecrosis and that the osteochondral grafting surgery replaced the damaged cartilage with plug cartilage that had greater superficial cartilage integrity and greater thickness parameter immediately after the surgery. Longterm effect of the osteochondral grafting surgery should be verified in further study.

Keywords Mosaicplasty · Ultrasound · Cartilage · Integrity · Thickness

\section{Introduction}

Osteochondral grafting surgery has been used for treatment of articular cartilage lesions [9, 10, 17, 25, 30, 31]. In this surgery, cylindrical plugs incorporating both articular cartilage and the underlying subchondral bone are harvested from the donor site and grafted to the lesion. Although this surgery replaces the damaged cartilage with the osteochondral plug [10] and several indentation studies measure cartilage mechanical stiffness during joint surgery [3, 22, 39], no human biomechanical study has compared the osteochondral plug cartilage with the damaged cartilage or adjacent healthy cartilage during the surgery yet. This may be because mechanical tests to measure stiffness take much time and are performed under mechanical compression that 
is a cause of plug subsidence, and therefore, are not ideal for use during the osteochondral grafting surgery.

High frequency pulse-echo ultrasound techniques have identified several characteristics of normal and degenerated articular cartilage $[1,5-7,11,12,14,15,18,22,27,28,33$, $34,37,38]$. The ultrasound signal strongly correlates with cartilage thickness $[15,36,37]$ and provides information about cartilage integrity $[1,5-7,37]$ and progression of osteoarthritis [14, 22, 28, 34, 38]. Recently, ultrasound techniques have been developed to measure surface fibrillation [7] and tissue thickness [15, 28, 37, 38], to assess cartilage integrity [14], to determine the effect of cartilage integrity on ultrasound backscatter [16, 34], for an ultrasonic indentation test [36] and for mechano-acoustic diagnosis [22]. High frequency ultrasound has been used for age-dependent assessment of articular cartilage $[5,16]$, and real-time analysis of articular cartilage degradation [33]. These studies have been suggested that ultrasound examination is sufficiently sensitive for determining structural properties [16, 22], roughness $[1,6,7]$ and thickness of cartilage [28, 36-38].

Especially, the high frequency pulse-echo ultrasound detects degeneration of the superficial collagen-rich cartilage zone [38] and microstructural changes in a superficial layer up to a depth of $500 \mu \mathrm{m}$ [13]. An ultrasound system provides information on integrity of cartilage and thickness of cartilage by wavelet transform [11, 18, 19, 27]. The wavelet transform can be performed under no mechanical compression to the cylindrical plugs, which are grafted by osteochondral grafting surgery, and therefore, can be used with no fear for plug subsidence during the surgery.

In this study, we hypothesized that the osteochondral grafting surgery replaced damaged cartilage of the International Cartilage Repair Society (ICRS) grade 3 with normal cartilage of osteochondral plugs and that the grade 3 cartilage showed lower signal intensity and the normal cartilage of osteochondral plugs showed higher signal intensity. In addition, we examined the parameter of thickness in the grade 3 cartilage, healthy-looking intact cartilage adjacent to the grade 3 cartilage and osteochondral plug cartilage using the ultrasound system. We also attempted to compare the two parameters in patients with traumatic cartilage lesions to those with osteonecrosis.

\section{Patients, materials and methods}

\section{Patients}

From August 2002 to November 2005, 37 patients with knee disorders who were attending the Department of Orthopaedic Surgery, Kyoto University Hospital, were screened for eligibility. The inclusion criteria were; (1) osteochondral grafting surgery, (2) focal traumatic cartilage lesion of the knee of the trochlea or femoral condyles, (3) osteonecrosis, (4) cartilage damage grade 3 (ICRS), (5) no upper age limit, and (6) informed consent. The exclusion criteria were; (1) osteochondritis dissecans, (2) early stage of osteoarthritis, (3) grade 1 and grade 2 cartilage lesions (ICRS). Here, ICRS describes cartilage standard evaluation form as follows: ICRS grade 0 , normal cartilage; grade 1, near-normal cartilage with superficial lesions; grade 2, cartilage with lesions extending to less than $50 \%$ of the depth of the cartilage; grade 3 , cartilage with defects that extend to more than $50 \%$ of the depth of the cartilage; grade 4 , severely abnormal cartilage that the cartilage defects reaches to subchondral bone (Table 1) [2]. Fifteen patients (mean age, 43.4 years; age range, 1461 years; 11 females and 4 males; 6 with traumatic cartilage lesions in the medial femoral condyle, 2 with traumatic cartilage lesions in the lateral femoral condyle, 3 with traumatic cartilage lesions in the patellar groove, and 4 with late stage of osteonecrosis in the medial femoral condyle) met with the criteria and participated in the ultrasound measurement (Table 2).

\section{Osteochondral grafting surgery}

The osteochondral grafting surgery was performed at 15 sites (two lateral and ten medial femoral condyles, and three patellar grooves) in 15 knees in the 15 patients. The mean interval between injury and surgery of the 15 patients was 8 months. Before surgery, the damaged cartilage was examined arthroscopically through standard anteromedial and anterolateral portals. Then, open surgery was performed via the parapatellar incision. The damaged cartilage and intact (healthy-looking) cartilage adjacent to the lesion apart $5-10 \mathrm{~mm}$ from the lesion were macroscopically assessed using the ICRS grading system. Then, ultrasound measurement, described below, of grade 3 of the damaged cartilage and the adjacent intact cartilage was performed. Followed by the measurement, the degenerated grade 3 damaged cartilage had been shaved until bleeding was observed from subchondral bone. This procedure of

Table 1 The ICRS grades

\begin{tabular}{ll}
\hline ICRS grades & Description \\
\hline Grade 0 & $\begin{array}{l}\text { Normal cartilage } \\
\text { Grade } 1\end{array}$ \\
Grade 2 & $\begin{array}{l}\text { Near-normal cartilage with superficial lesions } \\
\text { of the depth of the cartilage }\end{array}$ \\
Grade 3 & $\begin{array}{l}\text { Cartilage with defects that extend to more than } 50 \% \\
\text { of the depth of the cartilage } \\
\text { Grade } 4\end{array}$ \\
$\begin{array}{c}\text { Severely abnormal cartilage that the cartilage defects } \\
\text { reaches to subchondral bone }\end{array}$ \\
\hline
\end{tabular}


Table 2 Age, sex, cause of lesion, and area of the lesion for 15 patients

\begin{tabular}{lllll}
\hline Patients & Age & Sex & Traumatic cartilage lesion/osteonecrosis & Area of lesion \\
\hline No. 1 & 44 & Female & Traumatic cartilage lesion & Medial condyle \\
No. 2 & 14 & Female & Traumatic cartilage lesion & Lateral condyle \\
No. 3 & 46 & Male & Traumatic cartilage lesion & Patellar groove \\
No. 4 & 48 & Female & Traumatic cartilage lesion & Medial condyle \\
No. 5 & 49 & Female & Traumatic cartilage lesion & Medial condyle \\
No. 6 & 25 & Female & Traumatic cartilage lesion & Lateral condyle \\
No. 7 & 32 & Male & Traumatic cartilage lesion & Patellar groove \\
No. 8 & 46 & Male & Traumatic cartilage lesion & Medial condyle \\
No. 9 & 38 & Male & Traumatic cartilage lesion & Patellar groove \\
No. 10 & 50 & Female & Traumatic cartilage lesion & Medial condyle \\
No. 11 & 38 & Female & Traumatic cartilage lesion & Medial condyle \\
No. 12 & 55 & Female & Osteonecrosis & Medial condyle \\
No. 13 & 61 & Female & Osteonecrosis & Medial condyle \\
No. 14 & 53 & Female & Osteonecrosis & Medial condyle \\
No. 15 & 52 & Female & Osteonecrosis & Medial condyle \\
\hline
\end{tabular}

inducing bleeding is believed to accelerate healing of surrounding cartilage, and some evidence has been reported that the area is filled by newly formed reparative tissue $[25,29]$. After the shaving, a recipient hole was created in the shaved area with an osteochondral autograft transfer system (Arthrex, Naples, FL, USA). A dilator device was used to ensure that the tunnel was appropriate for the graft. An osteochondral plug was then harvested from a site medial or lateral to the patellar groove and grafted into the hole. These procedures were repeated until the shaved area was well covered by plugs (Fig. 1). In the 15 lesion sites in the 15 knees, 53 full-thickness osteochondral plugs were grafted. Mean number of plugs per lesion was 3.5, mean

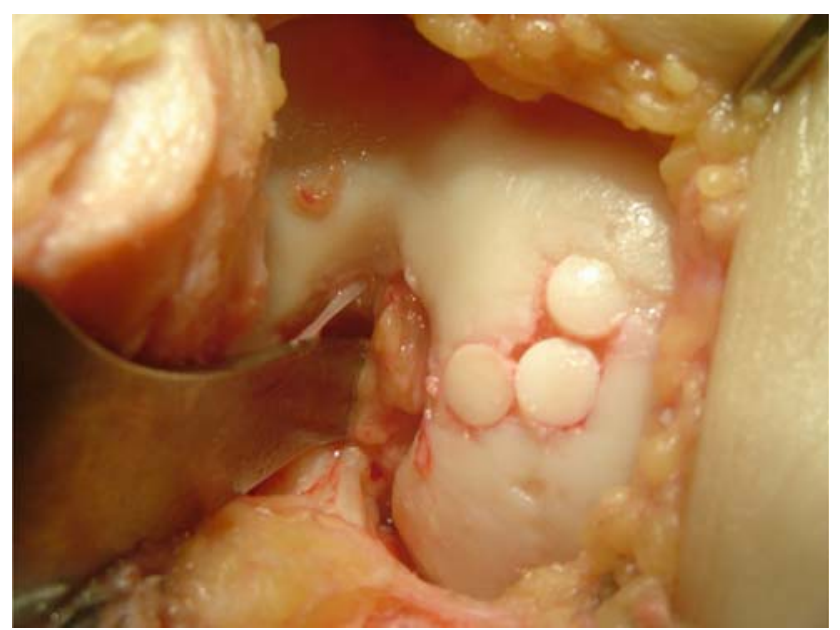

Fig. 1 Osteochondral grafting surgery. After the damaged cartilage of the medial condyle (ICRS grade 3 ) had been shaved, three plugs harvested from the donor site of the lateral patellar groove were grafted lesion size was $375 \mathrm{~mm}^{2}$, and mean plug size grafted was $7 \mathrm{~mm}$ in diameter.

\section{Ultrasound measurement}

The ultrasound measurement was performed at 31 points of the intact cartilage adjacent to the 15 lesion sites (mean; 2.0 points per lesion), 34 points of the grade 3 damaged cartilage of the 15 lesion sites (mean; 2.3 points per lesion) and 53 points of center of the 53 osteochondral plugs grafted to the 15 lesion sites. After the ICRS grading for the adjacent intact cartilage and the damaged cartilage had been done, signal intensity (a measure of superficial cartilage integrity) and interval between signals (i.e., time-offlight, which is related to thickness and ultrasound speed of cartilage) of the adjacent intact cartilage and the damaged cartilage were measured using an ultrasound system, which has been described previously [18-21, 27]. Briefly, the system consists of a transducer, a pulser/receiver (Model 5800PR; Panametrics Japan, Tokyo, Japan) and a personal computer (Fig. 2). The system can be set up for arthroscopic use, open surgery, or with a saline bath for experimental measurement (Fig. 2). The diameter of the transducer is approximately $3 \mathrm{~mm}$ and is covered with a saline-filled cone (Fig. 2). The ultrasound was set at $10 \mathrm{MHz}$ center frequency, using the immersion pulse-echo mode of the Model 5800PR, and the system was set up for open surgery. The signal intensity and the interval between signals of cartilage of the grafted osteochondral plugs were measured during the surgery.

Reproducibility of the system was confirmed by coefficient of variance. Twenty repeated measurements with the system set up as an experimental saline bath established the coefficients of variance were $0.9 \%$ for signal intensity, 


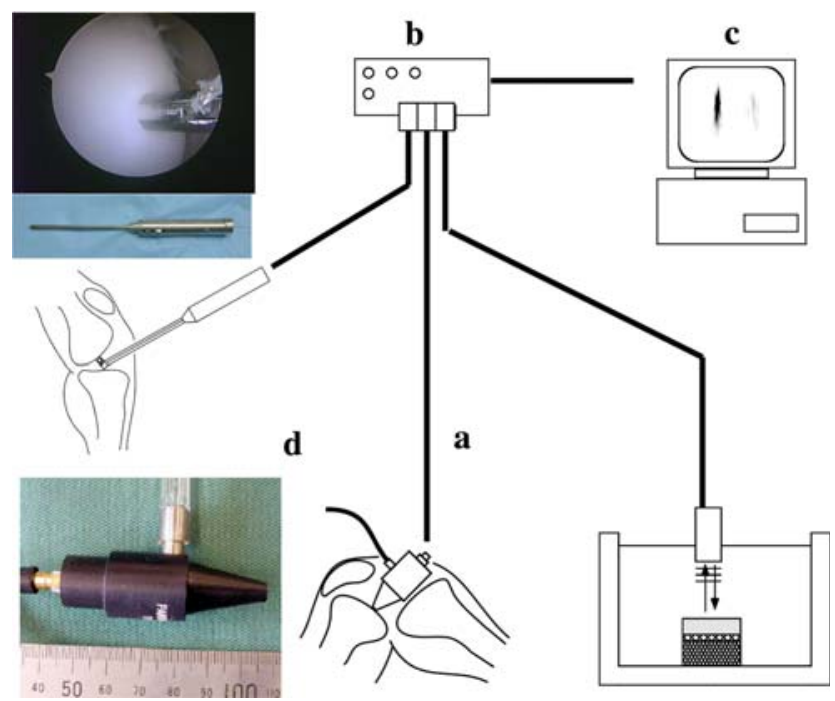

Fig. 2 The ultrasound measurement system consists of a transducer (a), a pulser/receiver (b), and a personal computer (c). The system can be set up for arthroscopic use, open surgery [real picture (d)], or with a saline bath for experimental measurement

$2.4 \%$ for signal duration, and $0.0 \%$ for the interval between signals.

\section{Statistical analysis}

After averaging the data from intact cartilage, damaged cartilage and the cartilage of the plugs in each of the 15 sites, statistical analysis for comparison among the intact cartilage, the damaged cartilage and the plug cartilage of the traumatic cartilage lesions was performed using the ANOVA and post hoc Scheffe's $F$-test. Statistical analysis for comparison among the intact cartilage, the damaged cartilage and the plug cartilage of the osteonecrosis was performed using the non-parametric Kruskal-Wallis test and the post hoc Scheffe's $F$-test. The $P$-values below 0.05 were seen as statistically significant.

\section{Results}

In the traumatic cartilage lesions, the damaged cartilage was macroscopically assessed as ICRS grade 3 (23 points of 11 lesion sites) and the intact cartilage as ICRS grade 0 (25 points adjacent to the 11 lesion sites). The signal intensities of ultrasound for the adjacent intact cartilage, the damaged cartilage, and the cartilage of plugs (37 points of the 37 plugs) were $1.91 \pm 1.75$ (relative value, arbitrary unit, mean $\pm \mathrm{SD}$ ), $0.40 \pm 0.37$, and $2.13 \pm 1.94$, respectively (Fig. 3a). The interval between signals of ultrasound for the adjacent intact cartilage, the damaged cartilage, and the cartilage of plugs were $2.79 \pm 0.67(\mu \mathrm{s}), 1.90 \pm 0.68$, and $2.85 \pm 0.56$, respectively (Fig. 3b). Statistical analysis
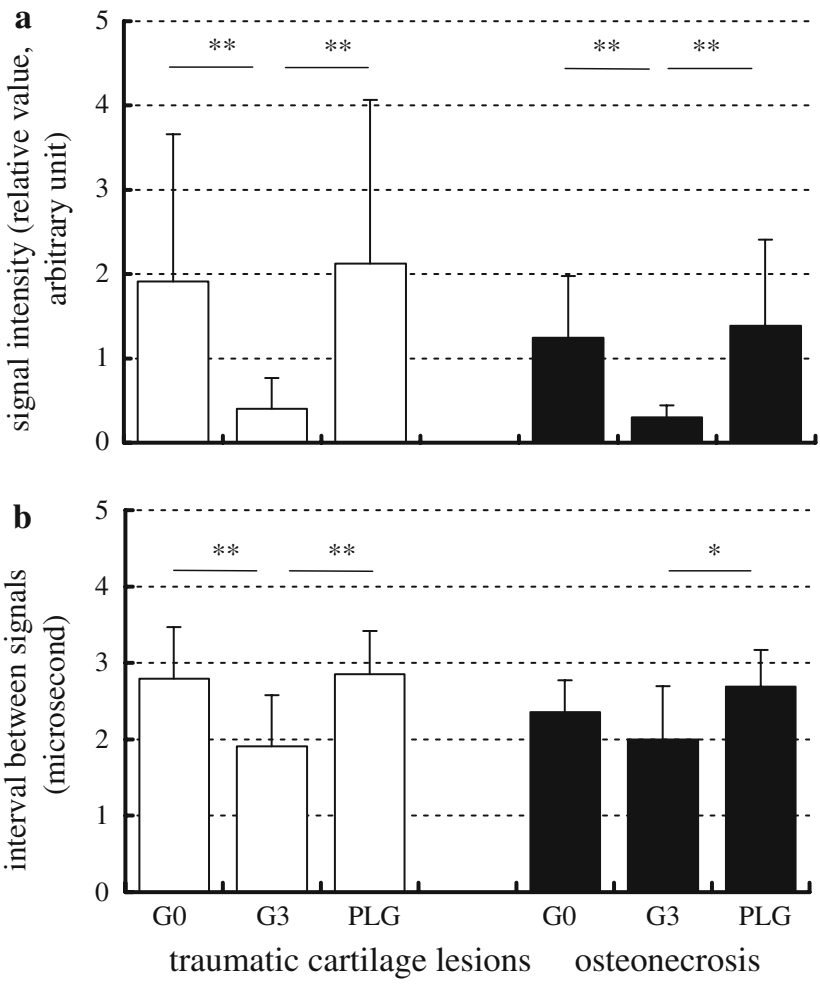

Fig. 3 Mean value and SD of adjacent intact cartilage (G0, grade 0), grade 3 cartilage (G3) and plug cartilage (PLG) of 15 knees. a Statistical analysis revealed significant differences in the signal intensity. The signal intensity of plug cartilage of cartilage lesion was $533 \%$ of the grade 3 cartilage and that of osteonecrosis was $463 \%$ of the grade 3 cartilage. b Significant differences were seen in the interval between signals. The interval between signals of plug cartilage of cartilage lesion was $150 \%$ of the grade 3 cartilage and that of osteonecrosis was $135 \%$ of the grade 3 cartilage. ${ }^{*} P<0.05$, $* * P<0.01$

revealed significant differences in the signal intensity (intact vs. damaged, $P<0.01$; damaged vs. plug, $P<0.01$ ) and in the interval between signals (intact vs. damaged, $P<0.01$; damaged vs. plug, $P<0.01$ ) (Fig. 3a, b).

In the osteonecrosis, the damaged cartilage was macroscopically assessed as ICRS grade 3 (11 points of four lesion sites) and intact cartilage as grade 0 (6 points adjacent to the four lesion sites). The signal intensities for the adjacent intact cartilage, the damaged cartilage, and the cartilage of plugs (16 points of the 16 plugs) were $1.25 \pm 0.73$ (relative value, arbitrary unit), $0.30 \pm 0.14$, and $1.39 \pm 1.02$, respectively (Fig. 3a). The interval between signals were $2.36 \pm 0.42(\mu \mathrm{s}), 2.00 \pm 0.69$, and $2.69 \pm 0.48$, respectively (Fig. 3b). Statistical analysis revealed significant differences in the signal intensity (intact vs. damaged, $P<0.01$; damaged vs. plug, $P<0.01$ ) and in the interval between signals (damaged vs. plug, $P<0.05$ ) (Fig. 3a, b).

Most of signal intensities in the damaged cartilage (29 of 34 measurement points) gathered below the value of 0.5 
(Fig. 4b). Although signal intensities in several intact cartilage ( 5 of 31 points) were below the 0.5 , the remaining signal intensities in intact cartilage (26 of 31 points) were greater than the 0.5 and were widely distributed from 0.52 to 7.30 (Fig. 4a). Signal intensities in a few points of plug cartilage ( 4 of 53 plugs) were lower than the value of the 0.5 and the remaining signal intensities in plug cartilage (49 of 53) were greater than value of the 0.5 (Fig. 4c). Although half of the interval between signals in damaged cartilage (17 of 34 points) were below $1.8 \mu$ s (Fig. 4b), all of the intervals between signals in the adjacent intact cartilage (31 of 31 ) and the plug cartilage (53 of 53) were over the $1.8 \mu$ s (Fig. $4 \mathrm{a}, \mathrm{c}$ ).

\section{Discussion}

High-frequency pulse-echo ultrasound is sensitive for detecting degeneration of the superficial collagen-rich cartilage zone [38]. Ultrasound measurements appear to be related to changes in the extracellular matrix collagen and fibrillar network organization [34]. Ultrasound can detect microstructural changes up to a depth of $500 \mu \mathrm{m}$ [13]. Therefore, signal intensity provides information on superficial collagen integrity of cartilage. In the present study, the signal intensities of the damaged cartilage in the traumatic cartilage lesions and in the osteonecrosis were $21 \%$ (0.40 vs. 1.91$)$ and $24 \%(0.30$ vs. 1.25$)$ of the adjacent intact cartilage, respectively (Fig. 3a). Lower signal intensity represents lower superficial collagen integrity of cartilage. Because it is well known that articular cartilage has limited intrinsic healing potential, lower signal intensity may be a critical sign of cartilage.

The signal intensities of plug cartilage in the traumatic cartilage lesions and in the osteonecrosis were 533\% (2.13 vs. 0.40$)$ and $463 \%$ (1.39 vs. 0.30$)$ of the damaged cartilage, respectively (Fig. 3a). Therefore, our results indicate that the osteochondral grafting surgery replaced the lesion with cartilage of greater signal intensity, which means greater superficial collagen integrity of cartilage, in addition, with cartilage of greater interval between signals, which means greater thickness of cartilage (Fig. 3b).

As seen in the damaged cartilage, decrease in the signal intensity below the value of 0.5 is one of the signs for cartilage damage (Fig. 4b). Several measurements in the signal intensities in healthy-looking intact cartilage were below the value of 0.5 (Fig. 4a), suggesting that very early event of osteoarthritis might occur in the macroscopically healthy-looking intact cartilage. It is well known that untreated cartilage damage will progress to osteoarthritis $[26,35]$. If no treatment was performed to the lesion, cartilage damage might spread over the adjacent intact cartilage from the damaged cartilage and progress to severe
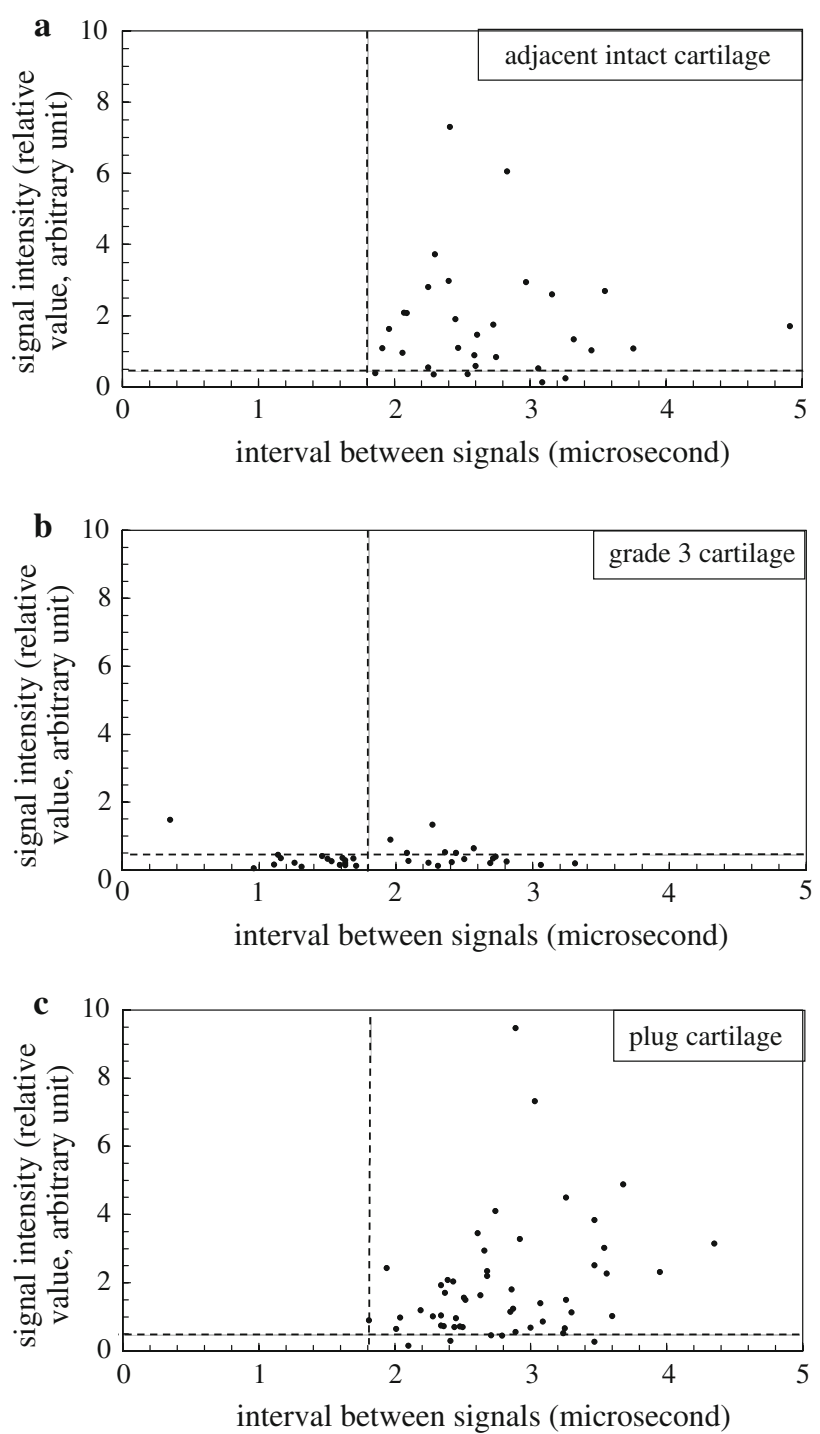

Fig. 4 The scattered graph of the signal intensity and the interval between signals. a Most of signal intensities in the adjacent healthylooking intact cartilage (26 of 31 measurement points, 84\%) were greater than the value of 0.5 . All of the intervals between signals ( 31 of $31,100 \%$ ) were over the value of $1.8 \mu$ s (dotted line). b Most of signal intensities of the grade 3 cartilage ( 29 of 34 points, $85 \%$ ) were lower than the value of 0.5 (dotted line). Half of the interval between signals (17 of $34,50 \%$ ) were below $1.8 \mu \mathrm{s}$. c Most of signal intensities in the plug cartilage ( 49 of $53,92 \%$ ) were greater than the value of 0.5 . All of the intervals between signals (53 of 53, 100\%) were over the $1.8 \mu$ s (dotted line)

arthritic conditions. The osteochondral grafting surgery contributes not only the damaged cartilage to be replaced with normal cartilage but also the adjacent intact cartilage not to progress to arthritic condition.

Two studies of a goat osteochondral grafting model revealed that mechanical stiffness of grafted cartilage had increased after the surgery, comparing with contralateral control site [23, 24]. The studies raise the question of whether osteochondral grafting causes these mechanical 
changes to the cartilage of osteochondral plugs [23, 24]. Mechanical measurement during the osteochondral grafting surgery is necessary to clarify this question. However, mechanical tests, such as indentation tests, are not ideal means because they are performed under mechanical compression. The mechanical compression causes osteochondral plugs to subside. Therefore, no human study has verified the mechanical features in cartilage of osteochondral plugs during the surgery yet. Ultrasound measurement can be performed under no mechanical compression with no fear of plug subsidence. Here, caution should be paid on this study because ultrasound signal intensity is not identical to mechanical stiffness of cartilage. Although mechanical measurement such as indentation tests of cartilage provides aggregate modulus of material properties, ultrasound measurement provides acoustic impedance, which is a function of density, Poisson's ratio, and Young's modulus of the material and structural properties of cartilage. Both mechanical and ultrasound measurements assess material and structural properties in different ways.

In the present study, signal intensity of cartilage of plugs was $12 \%$ greater than that of adjacent intact cartilage in the traumatic cartilage lesions (2.13 vs. 1.91) and $11 \%$ in the osteonecrosis (1.39 vs. 1.25$)$, but this difference was not significant. Therefore, the osteochondral grafting surgery has no harmful effect on human cartilage in signal intensity immediately after the surgery. In an animal study of rabbits, the signal intensity of plug cartilage of 8 and 12 weeks after the osteochondral grafting surgery was significantly lower than that of non-treated control cartilage but the signal intensity of the plug cartilage of 52 weeks after the surgery did not differ from that of the non-treated control cartilage [21]. Because the ultrasound measurement can be performed arthroscopically, therefore, postoperative change in the signal intensity of osteochondral plugs should be verified in second-look arthroscopy in human knees. This arthroscopic verification is ongoing.

In the present study, interval between signals in the damaged cartilage of grade 3 of trauma and osteonecrosis were $68 \%$ (1.90 vs. 2.79$)$ and $85 \%$ (2.00 vs. 2.36$)$ of the healthy-looking intact cartilage, respectively (Fig. 3b). In accordance with ICRS grading, grade 3 describes defects extending more than $50 \%$ of cartilage depth from the cartilage surface and grade 2 describes lesions extending less than $50 \%$ [2]. The values of the present study of 68 and $85 \%$ were same with 32 and $15 \%$ from cartilage surface, respectively, which classifies the cartilage as grade 2, indicates that the limitations of macroscopic assessment should be taken into account. If cartilage with a fissure is macroscopically diagnosed as grade 3 , ultrasound may pick this up using a pre-defined sound speed of grade 3 cartilage. For this purpose, it is needed to determine the sound speed of degraded grade 3 cartilage of human in further study. The value of $1.8 \mu$ s of the interval between signals corresponds to $1.44 \mathrm{~mm}$ thickness of cartilage (sound speed, $1,600 \mathrm{~m} / \mathrm{s}$ ). The intervals between signals in all of the healthy-looking intact cartilage (31 of 31) and all of the plug cartilage (53 of 53) were over the $1.8 \mu \mathrm{s}$. On the other hand, the intervals between signals in half of the damaged cartilage (17 of 34 ) were below the $1.8 \mu \mathrm{s}$. These findings indicate that cartilage thickness maintained in the adjacent intact cartilage and the plug cartilage but decreased in the damaged cartilage. In addition, sound speed might decrease in the damaged cartilage. Distribution in the signal intensity and the interval between signals represents the difference in ultrasound feature of the damaged cartilage (Fig. 4b). The distribution of the adjacent healthy-looking intact cartilage is almost same with that of the plug cartilage (Fig. 4a, c).

Clinically significant progress in preventing osteoarthritis depends on advances in understanding of the pathogenesis of osteoarthritis that will make it possible to decrease the risk of articular surface degeneration and facilitate articular surface repair and remodeling [4]. Ultrasound measurement is performed very short time within $0.5 \mathrm{~s}$ [20]. Thus, the ultrasound analysis contributes not only to detect the superficial deterioration of articular cartilage due to traumatic cartilage lesion or osteonecrosis but also to evaluate outcomes for surgical reconstruction, such as osteochondral grafting.

Hangody et al. have stated that 50 years of age constitutes the recommended upper limit for Mosaicplasty technique [8]. Nakagawa et al. have presented a report of five cases of age range 15-65 years [32]. In the report, preoperative International Knee Documentation Committee score (IKDC) of two cases aged 51 years and 65 years were 45.5 and 33.8, respectively, and postoperative IKDC of them were 100 (18 months after surgery) and 89.6 (13 months after surgery), respectively [32]. Because we experienced these two cases, no upper limit of age was included as inclusion criteria in this study. Long-term results of IKDC in patients over 50 years of age are still unknown.

To our knowledge, this is the first study to measure ultrasound signal intensity and interval between signals of damaged grade- 3 cartilage, adjacent intact cartilage and cartilage of plugs immediately after human osteochondral grafting surgery. Weak part of this study is small number of patients.

In conclusion, ultrasound properties of the damaged cartilage from trauma or osteonecrosis did not differ a lot especially in the late stages of osteonecrosis. The osteochondral grafting surgery replaced the damaged cartilage with osteochondral plugs that had greater superficial cartilage integrity and greater interval between signals immediately after the surgery. Further study should be 
performed because long-term effect of the osteochondral grafting surgery on ultrasound properties is unknown.

Acknowledgments This study was performed at the Department of Orthopaedic Surgery, Graduate School of Medicine, Kyoto University. This study was supported in part by a grant from the 'Grant-in-Aid for Scientific Research, Japan' and a grant from the 'New Energy and Industrial Technology Development Organization (NEDO)', Japan. Authors declare that this study complies with the current laws of Japan.

Open Access This article is distributed under the terms of the Creative Commons Attribution Noncommercial License which permits any noncommercial use, distribution, and reproduction in any medium, provided the original author(s) and source are credited.

\section{References}

1. Adler RS, Dedrick DK, Laing TJ, Chiang EH, Meyer CR, Bland PH, Rubin JM (1992) Quantitative assessment of cartilage surface roughness in osteoarthritis using high frequency ultrasound. Ultrasound Med Biol 18:51-58

2. Brittberg M, Peterson L (1998) Introduction to an articular cartilage classification. ICRS Newsl 1:8

3. Brommer H, Laasanen MS, Brama PA, van Weeren PR, Helminen HJ, Jurvelin JS (2006) In situ and ex vivo evaluation of an arthroscopic indentation instrument to estimate the health status of articular cartilage in the equine metacarpophalangeal joint. Vet Surg 35:259-266

4. Buckwalter JA, Brown TD (2004) Joint injury, repair, and remodeling: roles in post-traumatic osteoarthritis. Clin Orthop Relat Res 423:7-16

5. Cherin E, Saied A, Laugier P, Netter P, Berger G (1998) Evaluation of acoustical parameter sensitivity to age-related and osteoarthritic changes in articular cartilage using $50-\mathrm{MHz}$ ultrasound. Ultrasound Med Biol 24:341-354

6. Chiang EH, Adler RS, Meyer CR, Rubin JM, Dedrick DK, Laing TJ (1994) Quantitative assessment of surface roughness using backscattered ultrasound: the effects of finite surface curvature. Ultrasound Med Biol 20:123-135

7. Chiang EH, Laing TJ, Meyer CR, Boes JL, Rubin JM, Adler RS (1997) Ultrasonic characterization of in vitro osteoarthritic articular cartilage with validation by confocal microscopy. Ultrasound Med Biol 23:205-213

8. Hangody L, Fules P (2003) Autologous osteochondral mosaicplasty for the treatment of full-thickness defects of weightbearing joints: ten years of experimental and clinical experience. J Bone Joint Surg Am 85-A(Suppl 2):25-32

9. Hangody L, Kish G, Karpati Z, Eberhardt R (1997) Osteochondral plugs: autogenous osteochondral mosaicplasty for the treatment of focal chondral and osteochondral articular defects. Oper Tech Orthop 7:312-322

10. Hangody L, Kish G, Karpati Z, Szerb I, Udvarhelyi I (1997) Arthroscopic autogenous osteochondral mosaicplasty for the treatment of femoral condylar articular defects. A preliminary report. Knee Surg Sports Traumatol Arthrosc 5:262-267

11. Hattori K, Mori K, Habata T, Takakura Y, Ikeuchi K (2003) Measurement of the mechanical condition of articular cartilage with an ultrasonic probe: quantitative evaluation using wavelet transformation. Clin Biomech (Bristol, Avon) 18:553-557

12. Hattori K, Takakura Y, Morita Y, Takenaka M, Uematsu K, Ikeuchi K (2004) Can ultrasound predict histological findings in regenerated cartilage? Rheumatology (Oxford) 43:302-305
13. Hattori K, Takakura Y, Ohgushi H, Habata T, Uematsu K, Yamauchi J, Yamashita K, Fukuchi T, Sato M, Ikeuchi K (2005) Quantitative ultrasound can assess the regeneration process of tissue-engineered cartilage using a complex between adherent bone marrow cells and a three-dimensional scaffold. Arthritis Res Ther 7:R552-R559

14. Joiner GA, Bogoch ER, Pritzker KP, Buschmann MD, Chevrier A, Foster FS (2001) High frequency acoustic parameters of human and bovine articular cartilage following experimentallyinduced matrix degradation. Ultrason Imaging 23:106-116

15. Jurvelin JS, Rasanen T, Kolmonen P, Lyyra T (1995) Comparison of optical, needle probe and ultrasonic techniques for the measurement of articular cartilage thickness. J Biomech 28:231-235

16. Kim HK, Babyn PS, Harasiewicz KA, Gahunia HK, Pritzker KP, Foster FS (1995) Imaging of immature articular cartilage using ultrasound backscatter microscopy at $50 \mathrm{MHz}$. J Orthop Res 13:963-970

17. Kish G, Modis L, Hangody L (1999) Osteochondral mosaicplasty for the treatment of focal chondral and osteochondral lesions of the knee and talus in the athlete. Rationale, indications, techniques, and results. Clin Sports Med 18:45-66

18. Kuroki H, Nakagawa Y, Mori K, Ikeuchi K, Nakamura T (2004) Mechanical effects of autogenous osteochondral surgical grafting procedures and instrumentation on grafts of articular cartilage. Am J Sports Med 32:612-620

19. Kuroki H, Nakagawa Y, Mori K, Ohba M, Suzuki T, Mizuno Y, Ando K, Takenaka M, Ikeuchi K, Nakamura T (2004) Acoustic stiffness and change in plug cartilage over time after autologous osteochondral grafting: correlation between ultrasound signal intensity and histological score in a rabbit model. Arthritis Res Ther 6:R492-R504

20. Kuroki H, Nakagawa Y, Mori K, Kobayashi M, Yasura K, Okamoto Y, Mizuno Y, Ando K, Ikeuchi K, Nakamura T (2006) Maturation-dependent change and regional variations in acoustic stiffness of rabbit articular cartilage: an examination of the superficial collagen-rich zone of cartilage. Osteoarthritis Cartilage 14:784-792

21. Kuroki H, Nakagawa Y, Mori K, Kobayashi M, Okamoto Y, Yasura K, Nishitani K, Nakamura T (2007) Sequential changes in implanted cartilage after autologous osteochondral transplantation: postoperative acoustic properties up to 1 year in an in vivo rabbit model. Arthroscopy 23:647-654

22. Laasanen MS, Toyras J, Vasara AI, Hyttinen MM, Saarakkala S, Hirvonen J, Jurvelin JS, Kiviranta I (2003) Mechano-acoustic diagnosis of cartilage degeneration and repair. J Bone Joint Surg Am 85-A(Suppl 2):78-84

23. Lane JG, Tontz WL Jr, Ball ST, Massie JB, Chen AC, Bae WC, Amiel ME, Sah RL, Amiel D (2001) A morphologic, biochemical, and biomechanical assessment of short-term effects of osteochondral autograft plug transfer in an animal model. Arthroscopy 17:856-863

24. Lane JG, Massie JB, Ball ST, Amiel ME, Chen AC, Bae WC, Sah RL, Amiel D (2004) Follow-up of osteochondral plug transfers in a goat model: a 6-month study. Am J Sports Med 32:1440-1450

25. Matsusue Y, Yamamuro T, Hama H (1993) Arthroscopic multiple osteochondral transplantation to the chondral defect in the knee associated with anterior cruciate ligament disruption. Arthroscopy 9:318-321

26. Messner K, Maletius W (1996) The long-term prognosis for severe damage to weight-bearing cartilage in the knee: a 14-year clinical and radiographic follow-up in 28 young athletes. Acta Orthop Scand 67:165-168

27. Mori K, Hattori K, Habata T, Yamaoka S, Aoki H, Morita Y, Takakura Y, Tomita N, Ikeuchi K (2002) Measurement of the mechanical properties of regenerated articular cartilage using wavelet transformation. In: Ikada Y, Umakoshi Y, Hotta T (eds) 
Tissue engineering for therapeutic use, 6th edn. Elsevier, Tokyo, pp 133-142

28. Myers SL, Dines K, Brandt DA, Brandt KD, Albrecht ME (1995) Experimental assessment by high frequency ultrasound of articular cartilage thickness and osteoarthritic changes. J Rheumatol 22:109-116

29. Nakagawa Y, Matsusue Y, Ikeda N, Asada Y, Nakamura T (2001) Osteochondral grafting and arthroplasty for end-stage osteochondritis dissecans of the capitellum. A case report and review of the literature. Am J Sports Med 29:650-655

30. Nakagawa Y, Matsusue Y, Nakamura T (2003) Osteochondral graft transplantation for steroid-induced osteonecrosis of the femoral condyle. Lancet 362:402

31. Nakagawa Y, Matsusue Y, Suzuki T, Kuroki H, Nakamura T (2004) Osteochondral grafting for cartilage defects in the patellar grooves of bilateral knee joints. Arthroscopy 20(Suppl 2):32-38

32. Nakagawa Y, Suzuki T, Kuroki H, Kobayashi M, Okamoto Y, Nakamura T (2007) The effect of surface incongruity of grafted plugs in osteochondral grafting: a report of five cases. Knee Surg Sports Traumatol Arthrosc 15:591-596

33. Nieminen HJ, Toyras J, Rieppo J, Nieminen MT, Hirvonen J, Korhonen R, Jurvelin JS (2002) Real-time ultrasound analysis of articular cartilage degradation in vitro. Ultrasound Med Biol 28:519-525
34. Pellaumail B, Watrin A, Loeuille D, Netter P, Berger G, Laugier P, Saied A (2002) Effect of articular cartilage proteoglycan depletion on high frequency ultrasound backscatter. Osteoarthritis Cartilage 10:535-541

35. Sahlstrom A, Johnell O, Redlund-Johnell I (1997) The natural course of arthrosis of the knee. Clin Orthop Relat Res 340:152157

36. Suh JK, Youn I, Fu FH (2001) An in situ calibration of an ultrasound transducer: a potential application for an ultrasonic indentation test of articular cartilage. J Biomech 34:1347-1353

37. Toyras J, Lyyra-Laitinen T, Niinimaki M, Lindgren R, Nieminen MT, Kiviranta I, Jurvelin JS (2001) Estimation of the Young's modulus of articular cartilage using an arthroscopic indentation instrument and ultrasonic measurement of tissue thickness. J Biomech 34:251-256

38. Toyras J, Nieminen HJ, Laasanen MS, Nieminen MT, Korhonen RK, Rieppo J, Hirvonen J, Helminen HJ, Jurvelin JS (2002) Ultrasonic characterization of articular cartilage. Biorheology 39:161-169

39. Toyras J, Korhonen RK, Voutilainen T, Jurvelin JS, Lappalainen $\mathrm{R}$ (2005) Improvement of arthroscopic cartilage stiffness probe using amorphous diamond coating. J Biomed Mater Res B Appl Biomater 73:15-22 